\title{
Thermoresponsive $\mathrm{N}$-alkoxyalkylacrylamide polymers as a sieving matrix for high-resolution DNA separations on a microfluidic chip
}

\author{
Brian E. Root ${ }^{1}$, Mallory L. Hammock ${ }^{2}$, and Annelise E. Barron ${ }^{2, \dagger}$ \\ ${ }^{1}$ Department of Materials Science and Engineering, Northwestern University, Evanston, IL 60208 \\ ${ }^{2}$ Department of Chemical and Biological Engineering, Northwestern University, Evanston, IL \\ 60208
}

\section{Abstract}

In recent years, there has been an increasing demand for a wide range of DNA separations that require the development of materials to meet the needs of high resolution and high throughput. Here, we demonstrate the use of thermoresponsive $N$-alkoxyalkylacrylamide polymers as a sieving matrix for DNA separations on a microfluidic chip. The viscosities of the $N$ -

alkoxyalkylacrylamide polymers are more than an order of magnitude lower than that of a linear polyacrylamide of corresponding molecular weight, allowing rapid loading of the microchip. At $25^{\circ} \mathrm{C}, \mathrm{N}$-alkoxyalkylacrylamide polymers can provide improved DNA separations compared to LPA in terms of reduced separation time and increased separation efficiency, particularly for the larger DNA fragments. The improved separation efficiency in $N$-alkoxyalkylacrylamide polymers is attributed to the peak widths increasing only slightly with DNA fragment size, while the peak widths increase appreciably above $150 \mathrm{bp}$ using an LPA matrix. Upon elevating the temperature to $50{ }^{\circ} \mathrm{C}$, the increase in viscosity of the $N$-alkoxyalkylacrylamide solutions is dependent upon their overall degree of hydrophobicity. The most hydrophobic polymers exhibit an LCST below $50^{\circ} \mathrm{C}$, undergoing a coil-to-globule transition followed by chain aggregation. DNA separation efficiency at $50{ }^{\circ} \mathrm{C}$ therefore decreases significantly with increasing hydrophobic character of the polymers, and no separations were possible with solutions with an LCST below $50{ }^{\circ} \mathrm{C}$. The work reported here demonstrates the potential for this class of polymer to be used for applications such as PCR product and RFLP sizing, and provides insight into the effect of polymer hydrophobicity on DNA separations.

\section{Keywords}

DNA separation; microchip; thermoresponsive polymer

\section{Introduction}

Biological and biomedical sciences benefited greatly from the paradigm shift from slab gels to capillary arrays for DNA separations and sequencing, exemplified by the complete sequencing of the human genome ahead of schedule $[1,2]$. Progression to the microfluidic platform has allowed for more rapid separations, smaller device footprints, and integration

\footnotetext{
Corresponding Author: Annelise E. Barron, Associate Professor, Stanford University, Department of Bioengineering, 318 Campus Drive, Clark Center, Room S170, Stanford, CA 94305, Tel: (650)721-1151, Fax: (650)723-8544, aebarron@ stanford.edu.

$\dagger$ Present Address: Department of Bioengineering, Stanford University, Stanford, CA 94305

The authors declare no conflicts of interest.
} 
of sample preparation with analysis $[3,4]$. As the applications for DNA separations become more diverse, there is a continual need to develop the materials necessary to meet the demand for high-resolution and high-throughput analyses [5]. The polymer solutions used as the DNA sieving matrices play a central role in improving resolution as they are responsible for the mode of DNA migration, which contributes directly to the quality of the separation $[3,6,7]$.

The separation of DNA molecules in microchannels is typically achieved by the use of a replaceable solution of a high molar mass linear acrylamide-based polymer or a cellulosebased polymer. To achieve high-resolution separations of PCR products, the polymer chains must be entangled, which results in a large increase in solution viscosity [7]. This presents a practical limitation to the system as the concomitant increase in viscosity can require much higher pressures and longer times to load a microchannel. Since borosilicate glass chip devices cannot withstand the high pressures that fused silica capillaries do, the shift from the capillary to the microchip platform has created significant interest in polymer solutions that can be loaded rapidly with the application of minimal pressure, while still providing highresolution separations.

One strategy to overcome this challenge has been to use thermoresponsive polymer solutions to decouple the viscosity of the solution during loading from the viscosity during electrophoresis. Investigations into thermoresponsive polymer solutions have included using graft copolymers (e.g. poly ( $N$-isopropylacrylamide)-graft-poly(ethylene oxide), or PNIPAg-PEO [8], and poly(acrylamide)-graft-poly( $N$-isopropylacrylamide) [9]) to separate dsDNA and Pluronic ${ }^{\mathrm{TM}}$ triblock copolymers to separate short oligonucleotides [10]. The studies that were done using PNIPA-g-PEO found that adequate sieving performance was only achieved with a high density of grafted PEO, and that sieving performance decreased above the solution's phase transition [8].

$N$-alkoxyalkylacrylamide homo- and co-polymers have been investigated as DNA sequencing matrices in capillaries [11]. Poly $(N$-ethoxyethylacrylamide) (pNEEA) was shown to exhibit a dramatic increase in viscosity above $36^{\circ} \mathrm{C}$, while poly $(N-$ methoxyethylacrylamide) (pNMEA) showed only a small increase in viscosity upon heating to $50^{\circ} \mathrm{C}$. Wada et al. have reported a "re-entrant"-type thermal response for gels of pNEEA, meaning that the polymer chains are in a swollen state at both low and high temperature, and in a shrunken state at intermediate temperatures [12]. This re-swelling at high temperature was hypothesized by Kan et al. [11] to provide a highly entangled solution through which the DNA could migrate. However, the pNEEA polymer solution resulted in poor sequencing results, while sequencing was successfully achieved, with a relatively long read, with pNMEA.

In this report, we provide a detailed physical characterization of a library of $N$ alkoxyalkylacrylamide homo- and co-polymers, and demonstrate the ability of this class of polymers to act as a high-resolution sieving matrix for PCR and RFLP sizing of doublestranded DNA molecules. The use of random copolymers provides an easier synthetic pathway to a final sieving polymer than the graft copolymers reported by other groups. In comparison to a previous report using this class of polymers [11], this study provides a more complete understanding of the thermoresponsive nature of the polymers in a nondenaturing buffer, compares dsDNA separations of $N$-alkoxyalkylacrylamide polymers with conventional LPA both below and above the transition temperature of the polymers in a microfluidic chip, and finally demonstrates that high NMEA-content matrices provide better separations than LPA, which may be due to an underlying difference in separation mechanism. 


\section{Materials and Methods}

\subsection{Polymer Synthesis}

Homopolymers and copolymers of $N$-methoxyethylacrylamide (NMEA) and $N$ ethyoxyethylacrylamide (NEEA) were synthesized via free-radical polymerization. Nomenclature for NMEA and NEEA copolymers gives the abbreviation for the monomer with its percent of total monomer by weight (e.g. the NMEA90-NEEA10 polymer was $90 \%$ NMEA monomer by weight and 10\% NEEA monomer of the total monomer in the reaction). Ultrapure NMEA and NEEA monomer (Monomer-Polymer and Dajac Labs, Feasterville, PA) were dissolved at $1 \% \mathrm{w} / \mathrm{v}$ total monomer concentration in water in a jacketed flask connected to a recirculating water bath at $25^{\circ} \mathrm{C}$. The solution was bubbled with nitrogen for 45 minutes prior to being initiated with $0.5 \mu$ of $10 \%(w / v)$ ammonium persulfate (APS) / ml reaction volume and $0.1 \mu \mathrm{l}$ of $N, N, N^{\prime}, N^{\prime}-$

tetramethylethylenediamine (TEMED)/ $\mathrm{ml}$ reaction volume (Sigma Aldrich, St. Louis, MO). Following a four-hour reaction, the solution was poured into 100,000 Da molecular weight cut-off cellulose acetate dialysis membranes (Spectrum Labs, Gardena, CA) and dialyzed against deionized water for at least 10 days with frequent water changes. Linear polymer acrylamide (LPA) and poly $\left(N\right.$-hydroxyethylacrylamide) (pHEA) were synthesized at $50{ }^{\circ} \mathrm{C}$ at $3 \% \mathrm{w} / \mathrm{v}$ and $1 \% \mathrm{w} / \mathrm{v}$ monomer concentration, respectively, and initiated with $4,4^{\prime}-$ azobis(4-cyanovaleric acid) (Sigma Aldrich, St. Louis, MO). The solutions were then frozen, lyophilized, and stored dry.

\subsection{Gel permeation chromatography - Multiple angle laser light scattering}

Polymers were analyzed by tandem gel permeation chromatography (GPC) - multiple angle laser light scattering (MALLS) using a Waters 2690 Alliance Separations Module with an on-line refractometer as previously described [13, 14]. Briefly, the polymer sample was dissolved at $1 \mathrm{mg} / \mathrm{ml}$ in the mobile phase of $100 \mathrm{mM} \mathrm{NaCl}, 50 \mathrm{mM} \mathrm{NaH}_{2} \mathrm{PO}_{4}$, and $200 \mathrm{ppm}$ $\mathrm{NaN}_{3}$. This solution was injected into the system and fractionated by three Shodex (New York, NY) OHpak columns (SB-806 HQ, SB-804 HQ, and SB-802.5 HQ) connected in series. The effluent from the GPC flows directly into a DAWN DSP Laser Photometer and Optilab DSP Interferometric Refractometer connected in series (both, Wyatt Technology, Santa Barbara, CA). The data is processed using ASTRA software from Wyatt Technology.

\subsection{Absorbance}

Absorbance at $500 \mathrm{~nm}$ was measured as a function of temperature to determine the approximate lower critical solution temperatures (LCSTs) of the various polymer solutions [15]. The polymers were dissolved at 5\% w/w in 1xTTE buffer (49 mM Trix, $49 \mathrm{mM}$ TAP, 2 mM EDTA) and these solutions were characterized on a Cary 500 (Varian, Palo Alto, CA). The instrument was run in double beam mode with $1 \mathrm{x}$ TTE buffer in the reference vial. The temperature was increased at $1{ }^{\circ} \mathrm{C} / \mathrm{min}$ and a data point was taken every $0.25{ }^{\circ} \mathrm{C}$.

\subsection{Rheology}

Rheological properties of the polymers were tested on an Anton Paar Physica MCR 300 rheometer (Ashland, VA, USA). A Peltier temperature controller connected to a recirculating water bath (Julabo USA Inc., Allentown, PA, USA) was used to provide the temperature control. Tests were performed with a cone-and-plate fixture (model CP50-1). Oscillation tests were performed at $0.1 \mathrm{~Pa}$ with an angular frequency of $1 \mathrm{~s}^{-1}$ and data points recorded every $1{ }^{\circ} \mathrm{C}$ during the temperature ramp. 


\subsection{Dynamic Light Scattering}

Dynamic light scattering (Beckman Coulter, Fullerton, CA) was used to characterize the approximate polymer coil size as a function of temperature. Polymer samples were dissolved at $0.5 \mathrm{mg} / \mathrm{ml}$ in distilled water and pushed through a $0.45 \mu \mathrm{m}$ filter (GE, Piscataway, NJ) to remove any particulates. The sample was given 5 minutes to equilibrate at each temperature and three data sets were taken at each temperature with data taken over two minutes. The data was fit to a unimodal distribution using a size distribution processor running CONTIN [15].

\subsection{DNA Separations}

Microchip electrophoresis experiments were performed using a custom-built laser-induced fluorescence and power supply system previously described [14]. DNA separations were performed using $5 \% \mathrm{w} / \mathrm{w}$ polymer solutions dissolved in 1xTTE with $5 \mu \mathrm{M} \mathrm{EtBr}$ (Fisher Scientific, Pittsburgh, PA). Separations were performed in glass microchips (Micronit, Enschede, The Netherlands) with a $7.5 \mathrm{~cm}$ effective separation distance and a field strength of $250 \mathrm{~V} / \mathrm{cm}$. The chips were dynamically coated using a pHEA solution as previous described [16]. The pBR322-MspI dsDNA digest (New England Biolabs, Ipswich, MA) at a concentration of $1 \mu \mathrm{g} / \mathrm{ml}$ was separated and the traces were analyzed using PeakFit (SYSTAT, Chicago, IL).

\section{Results and Discussion}

The molar mass, z-average radius of gyration, and polydispersity index of the polymers determined by tandem GPC-MALLS are shown in Table 1. The polymers were synthesized to have similar molecular weights so that differences in solution properties (e.g. viscoelastic and thermoresponsive behavior) and DNA separation performance are due to polymer chemistry with minimal impact from differences in average molecular weight. Poly- $N$ methoxyethylacrylamide (pNMEA) is the most hydrophilic thermoresponsive polymer investigated here, while poly- $N$-ethoxyethylacrylamide (pNEEA) is the least hydrophilic. While the monomers only differ by a single methyl group, the NMEA-NEEA composition is shown to have a significant effect on both the polymer solution properties and thermoresponsive behavior.

The viscoelastic and thermoresponsive behaviors of the polymers were investigated by absorbance, rheology, and light scattering. Turbidity measurements provide a method to determine if a solution exhibits a lower critical solution temperature (LCST), which is indicative of a dramatic, thermally induced volume phase transition of the polymers in solution [15]. Figure 2 shows that NEEA, NMEA10-NEEA90 and NMEA25-NEEA75 solutions all show LCSTs in the temperature range tested. The temperature at which the turbidity begins to increase in these solutions corresponds very closely with the sharp increase in complex viscosity shown in Figure 3. Therefore, the increase in viscosity seen for these polymers is likely due to the polymer coils undergoing a coil-to-globule transition followed by aggregation and phase separation [15]. Figure 3 also shows that the solutions that do not undergo an LCST increase in viscosity at elevated temperature. However, the viscosity increase of these solutions is not as sharp nor as pronounced as the solutions that undergo the LCST. DLS shows that the chain size of these polymers shrinks with increasing temperature (Figure 4). Therefore, while these solutions are not undergoing a phase transition, the increase in viscosity of these solutions is attributed to increased hydrophobic chain interactions.

Electrophoresis of a dsDNA digest through a 5\% (w/w) solution of each of these matrices demonstrates the potential application of this class of polymers, as well as limitations on the 
hydrophobicity of the sieving polymer. The viscosities at room temperature, Figure 3, of the $N$-alkoxyalkylacrylamide polymer solutions are significantly lower than that of LPA, allowing for rapid, facile loading and unloading of these solutions into the microfluidic chips. Figure 5 shows the separation efficiency of each of the polymer solutions at $25^{\circ} \mathrm{C}$. LPA is used as a reference due to its non-thermoresponsive behavior and its ubiquitous use in the field of DNA separations by microchannel electrophoresis. For DNA fragment sizes below approximately $150 \mathrm{bp}$, there is no significant difference in separation efficiency between LPA and $N$-alkoxylalkylacrylamide polymers with NMEA compositions greater than 50\%. Around $150 \mathrm{bp}$, the separation efficiency of the LPA matrix begins to drop, and with fragments having sizes larger than $200 \mathrm{bp}$, the separation efficiency of the NMEANEEA polymers is much greater than LPA. The three polymers with greater than 50\% NEEA composition provide separation efficiencies lower than that of LPA at all DNA fragment sizes, and in particular, separations of the larger DNA fragments are significantly worse than in LPA.

The improvement in dsDNA separation efficiency using the high-NMEA content polymers can be attributed to smaller peak widths, particularly for the larger DNA fragment sizes. Figure 6a shows the full width at half-maximum (FWHM) of the DNA peaks for each of the different polymer solutions. The FWHM for small DNA fragments is roughly equal for all polymer types (except for NMEA10-NEEA90). Comparing again the high-NMEA content polymers to LPA, the FWHM for LPA begins to increase around $150 \mathrm{bp}$ leading to a significant difference in peak width for fragments that are $200 \mathrm{bp}$ and longer. This peak broadening corresponds with the drop in separation efficiency. Separations with the highNEEA content polymers resulted in peaks widths that were comparatively large. This is attributed to the greater hydrophobicity of the polymer solutions, resulting in an increase in polymer-DNA interactions.

DNA mobilities are much higher in the $N$-alkoxyalkylacrylamide polymers than in LPA (Figure S 1), resulting in shorter separation times. Lower mobilities may provide the DNA with more time to diffuse during the LPA-based separations, which may lead to wider peaks [3]. However, Figure $6 \mathrm{~b}$ shows the FWHM normalized to the peak migration time to account for this difference. Again, there appears to be a distinct DNA size at which the peak widths increase when using LPA, but not when using the high-NMEA content polymers. Differences in polymer architecture and chain entanglement can lead to differences in the migration mechanism of DNA [6, 7]. It has also been reported that a polymer matrix with an intermediate strength of entanglement can provide improved sequencing results as compared to a strongly entangled LPA solution [3]. Figure 3 shows that the $N$-alkoxyalkylacrylamide polymers do not provide an entangled network as strong as that of LPA, resulting in a lower polymer solution viscosity. It is possible that the high-NMEA content polymer solutions investigated here provide similar intermediate strengths of entanglement and minimal polymer hydrophobicity, which together result in smaller peak widths and improved separations for dsDNA.

Figure 7 shows a representative electropherogram of a DNA separation using LPA and NMEA. The figure shows a 20\% reduction in separation time using the NMEA solution. The peaks observed when using NMEA appear to be more Gaussian in shape, compared with the LPA traces that appear to exhibit more peak tailing. The middle region of the traces is expanded in the figure to show the importance of the higher separation efficiency that is achieved using NMEA. The arrows in the expanded view point to the $147 \mathrm{bp}$ doublet that is baseline-resolved using NMEA, but only partially resolved using LPA. The peaks of the larger fragments are also clearly sharper with the NMEA polymer as expected based on the FWHM data shown in Figure 6. These differences can be of key importance for PCR 
fragment or restriction fragment sizing where accurate determination of the DNA length is critical.

It is of interest here to note that the optimal polymer solution concentration may vary with the NMEA content of the polymer. Increasing the NMEA content increases the radius of gyration, as shown in Table 1. A larger radius of gyration should decrease the polymer overlap threshold concentration, $C^{*}$, as well as the polymer network entanglement concentration, $C_{e}$, as described by Chiesl et al. [7]. The mode of DNA migration through a polymer network is function of the polymer solution concentration with respect to these characteristic concentrations ( $C^{*}$ and $C_{e}$ ) that will differ for each polymer. Therefore, future work on investigating a range of polymer solution concentrations for the different polymer compositions can provide useful information on the optimal polymer composition and concentration for separating a specific size range of DNA.

DNA separations were also performed at $50{ }^{\circ} \mathrm{C}$ to gain insight into the effects of temperature on the ability of these thermoresponsive polymers to separate DNA. Figure 8 shows the separation efficiency for solutions of LPA, NMEA and NMEA90-NEEA10 at $50{ }^{\circ} \mathrm{C}$. Polymers with higher NEEA content either resulted in poor separations in which the DNA peaks significantly overlapped, or the solutions underwent an LCST and no peaks could be detected. The poor separation performance at $50{ }^{\circ} \mathrm{C}$ is believed to be due to hydrophobic interactions between the polymer chains and DNA resulting in broad peaks. The hydrophobic content of the polymer chains has a much greater impact at elevated temperatures, even for polymers that do not possess an LCST, as shown in Figure 4. Additionally, the difference in the separation efficiencies provided by NMEA and NMEA90-NEEA10 show that the small increase in hydrophobe content that did not significantly impact DNA separations at $25^{\circ} \mathrm{C}$ is highly detrimental to separations carried out at $50{ }^{\circ} \mathrm{C}$. Therefore, while the high-NMEA content polymers reported here provide an excellent sieving matrix for dsDNA at $25^{\circ} \mathrm{C}$, further tuning of the polymer chain architecture to achieve the balance between entanglement strength and polymer hydrophobicity may be necessary to use this class of polymers for higher temperature DNA separations such as STR sizing and DNA sequencing.

\section{Conclusions}

We have demonstrated the ability of $N$-alkoxyalkylacrylamide polymers to provide enhanced dsDNA separations compared to LPA at $25^{\circ} \mathrm{C}$. This improvement when using high-NMEA content polymers is attributed to smaller peak widths, particularly for DNA molecules longer than $150 \mathrm{bp}$, even when peak widths are normalized with the migration time. Electropherograms of separations performed at $25^{\circ} \mathrm{C}$ show that the increase in separation efficiency using an NMEA polymer can provide baseline resolution of certain DNA fragments that is not achieved with the LPA matrix. The improvement in separation efficiency, reduction in separation time, and ease of loading the $N$-alkoxyalkylacrylamide solutions reported here make them an excellent matrix for microchip separation applications such as PCR or RFLP sizing.

While the NMEA homopolymers provided a high-resolution separation at $50{ }^{\circ} \mathrm{C}$, the separation efficiencies for co-polymers of NMEA and NEEA were much more sensitive to NEEA content than at $25^{\circ} \mathrm{C}$. Additionally, higher NEEA content polymers exhibit an LCST below $50{ }^{\circ} \mathrm{C}$, which prevents successful dsDNA separations. Tuning of the polymer matrix to balance the strength of entanglement and degree of hydrophocity at higher temperatures similar to may allow this class of polymers to provide enhanced separations for applications such as sequencing similar what was reported here at $25^{\circ} \mathrm{C}$ for dsDNA separations. 


\section{Supplementary Material}

Refer to Web version on PubMed Central for supplementary material.

\section{Acknowledgments}

Financial support was provided by the NSF through the Northwestern University Nanoscale Science and Engineering Center (Award No. EEC-0647560) and the NIH (Grant No. 1 U01 AI061297-02 and 5R01HG003583 via Microchip Biotechnologies, Inc.). Brian Root was supported while on appointment as a U.S. Department of Homeland Security (DHS) Fellow under the DHS Scholarship and Fellowship Program, a program administered by the Oak Ridge Institute for Science and Education (ORISE) for DHS through an interagency agreement with the U.S Department of Energy (DOE). ORISE is managed by Oak Ridge Associated Universities under DOE contract number DE-AC05-06OR23100. All opinions expressed in this paper are the author's and do not necessarily reflect the policies and views of DHS, DOE, NSF, NIH or ORISE. This work was also supported by grant \# 2 R01 HG001970-07 from the National Human Genome Research Institute of the National Institutes of Health. However, the views expressed in this paper are solely the responsibility of the authors and do not necessarily represent the official views of the National Human Genome Research Institute or the National Institutes of Health. The authors would like to thank Prof. Phil Messersmith in Biomedical Engineering at Northwestern University for use of the Beckman-Coulter Dynamic Light Scatter instrument and Northwestern University's Keck Biophysics Facility for use of the Cary 500.

\section{Abbreviations}

LPA linear polyacrylamide

pHEA $\quad \operatorname{poly}(\boldsymbol{N}$-hydroxyethylacrylamide $)$

pNEEA $\quad \operatorname{poly}(N$-ethoxyethylacrylamide)

pNMEA $\operatorname{poly}(N$-methoxyethylacrylamide $)$

PNIPA $\operatorname{poly}(N$-isopropylacrylamide

PEO poly(ethylene oxide)

GPC gel permeation chromatography

MALLS multiple angle laser light scattering

$\mathbf{M}_{\mathbf{w}} \quad$ weight-average molar mass

PDI polydispersity index

$\mathbf{R}_{\mathbf{z}} \quad$ z-average radius of gyration

FWHM full-width at half maximum

PCR polymerase chain reaction

RFLP restriction fragment length polymorphism

LCST lower critical solution temperature

DLS dynamic light scattering

\section{References}

1. Venter JC, Adams MD, Myers EW, Li PW, et al. Science. 2001; 291:1304-1351. [PubMed: 11181995]

2. Lander ES, Linton LM, Birren B, Nusbaum C, et al. Nature. 2001; 409:860-921. [PubMed: 11237011]

3. Fredlake CP, Hert DG, Kan CW, Chiesl TN, et al. Proc. Natl. Acad. Sci. USA. 2008; 105:476-481. [PubMed: 18184818] 
4. Easley CJ, Karlinsey JM, Bienvenue JM, Legendre LA, et al. Proc. Natl. Acad. Sci. USA. 2006; 103:19272-19277. [PubMed: 17159153]

5. Kan CW, Fredlake CP, Doherty EAS, Barron AE. Electrophoresis. 2004; 25:3564-3588. [PubMed: 15565709]

6. Chies1 TN, Putz KW, Babu M, Mathias P, et al. Anal. Chem. 2006; 78:4409-4415. [PubMed: 16808448]

7. Chiesl TN, Forster RE, Root BE, Larkin M, Barron AE. Anal. Chem. 2007; 79:7740-7747. [PubMed: 17874850]

8. Liang DH, Zhou SQ, Song LG, Zaitsev VS, Chu B. Macromolecules. 1999; 32:6326-6332.

9. Sudor J, Barbier V, Thirot S, Godfrin D, et al. Electrophoresis. 2001; 22:720-728. [PubMed: 11296927]

10. Zhang J, Gassmann M, He WD, Wan F, Chu B. Lab Chip. 2006; 6:526-533. [PubMed: 16572215]

11. Kan CW, Doherty EAS, Barron AE. Electrophoresis. 2003; 24:4161-4169. [PubMed: 14679563]

12. Wada N, Yagi Y, Inomata H, Saito S. Macromolecules. 1992; 25:7220-7222.

13. Buchholz BA, Doherty EAS, Albarghouthi MN, Bogdan FM, et al. Anal. Chem. 2001; 73:157164. [PubMed: 11199960]

14. Chiesl TN, Shi W, Barron AE. Anal. Chem. 2005; 77:772-779. [PubMed: 15679343]

15. Lessard DG, Ousalem M, Zhu XX, Eisenberg A, Carreau PJ. J. Polym. Sci. Pt. B-Polym. Phys. 2003; 41:1627-1637.

16. Albarghouthi MN, Buchholz BA, Huiberts PJ, Stein TM, Barron AE. Electrophoresis. 2002; 23:1429-1440. [PubMed: 12116153] 

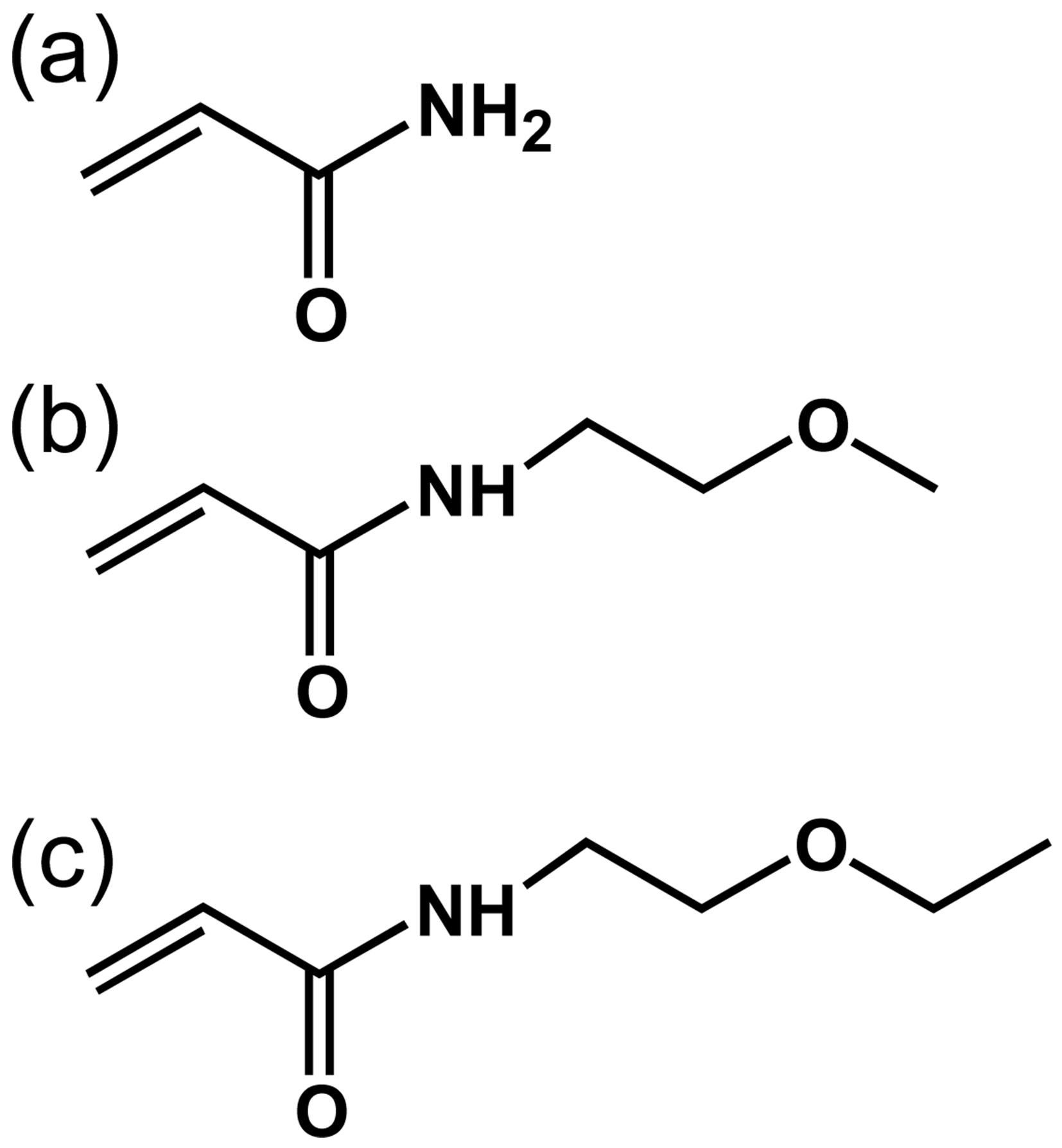

Figure 1.

Monomer structures: (a) acrylamide, (b) $\mathrm{N}$-methoxyethylacrylamide, and (c) $\mathrm{N}$ ethoxyethylacrylamide. 


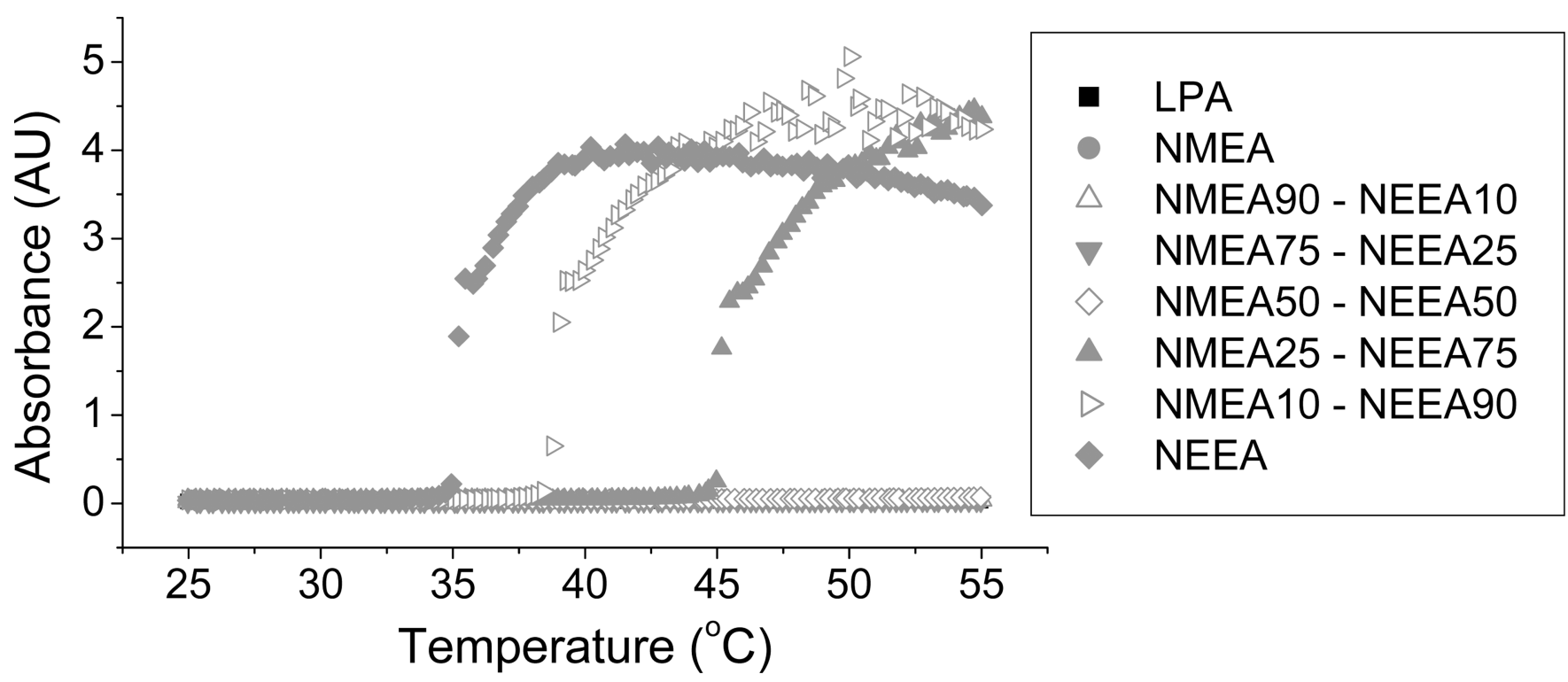

Figure 2.

Absorbance at $500 \mathrm{~nm}$ versus temperature for LPA, NMEA, and NEEA polymers. Only four data sets readily seen as data sets of polymers that do not possess an LCST overlay.

Temperature ramp of $1{ }^{\circ} \mathrm{C} / \mathrm{sec}$. 


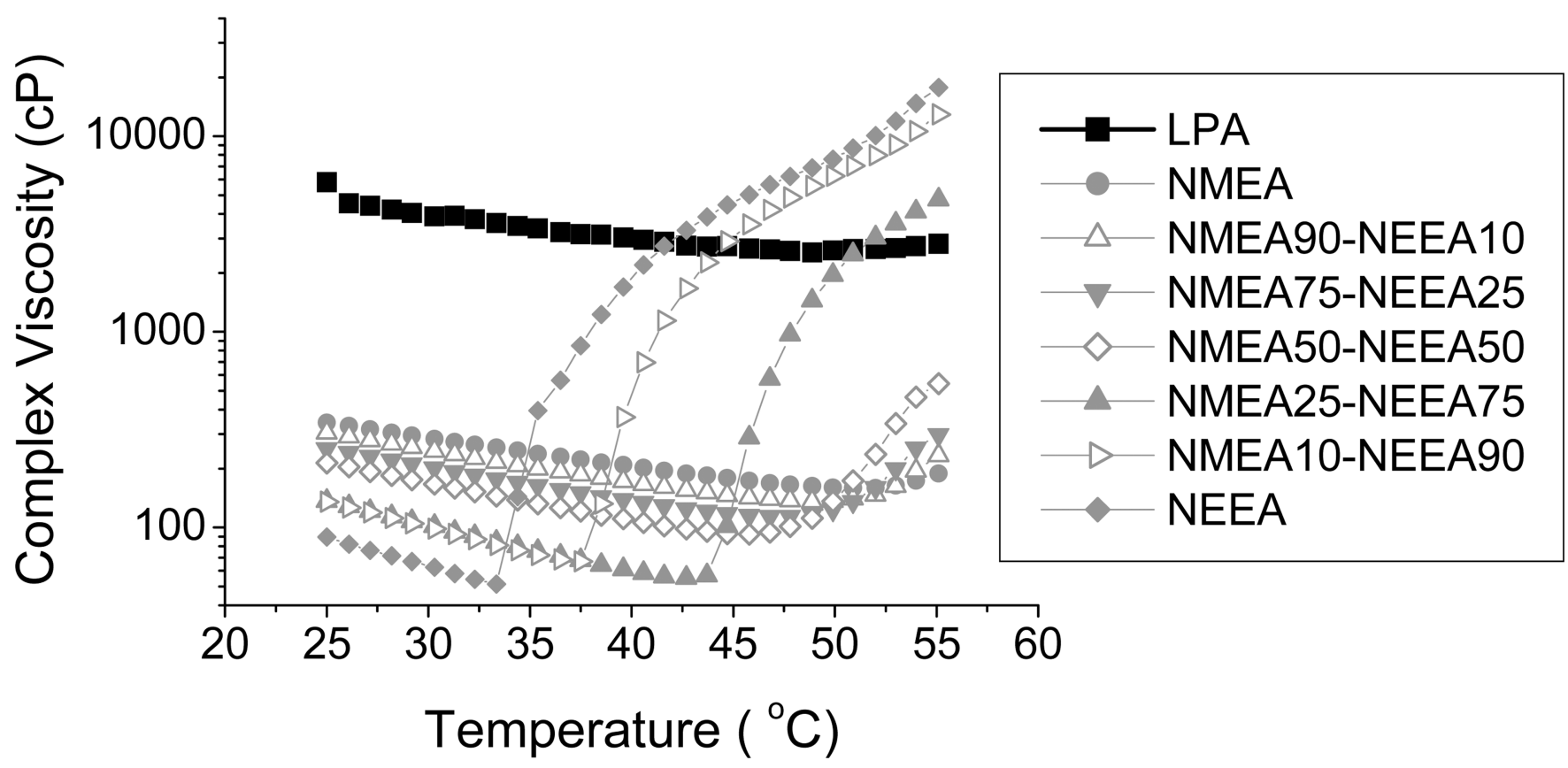

Figure 3.

Complex viscosity versus temperature for $5 \%(\mathrm{w} / \mathrm{w})$ polymer solutions. Oscillations at 1 $\mathrm{sec}^{-1}$ and $0.1 \mathrm{~Pa}$ with a temperature ramp of $2{ }^{\circ} \mathrm{C} / \mathrm{sec}$. $(\mathrm{n}=3)$ 


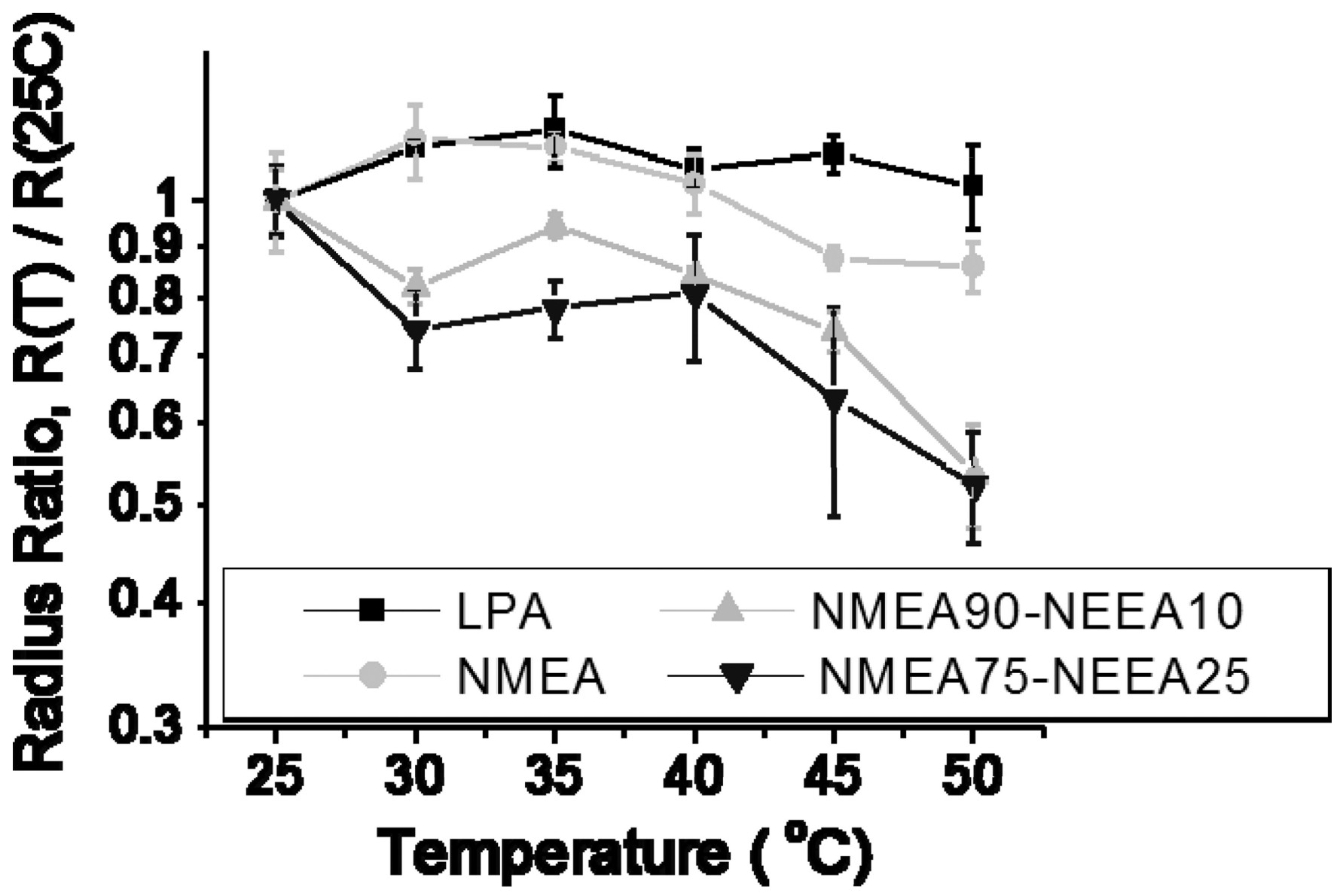

Figure 4.

Polymer hydrodynamic radius measured by DLS at multiple temperatures normalized to the average hydrodynamic radius at $25^{\circ} \mathrm{C}$. $(\mathrm{n}=3)$ 


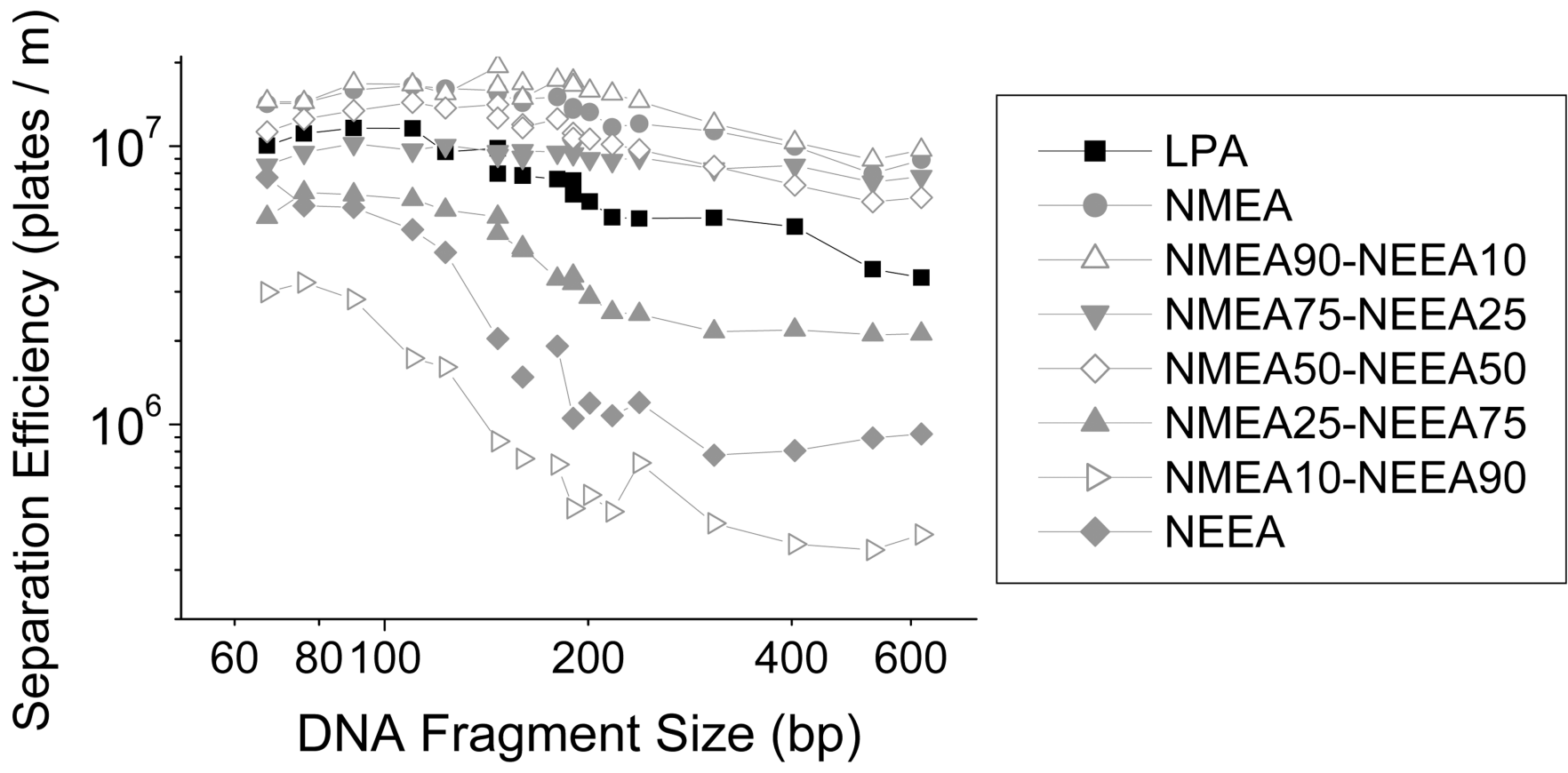

Figure 5.

Average separation efficiency of $5 \%(\mathrm{w} / \mathrm{w})$ polymer solutions at $25^{\circ} \mathrm{C}$. $(\mathrm{n}=3)$ 

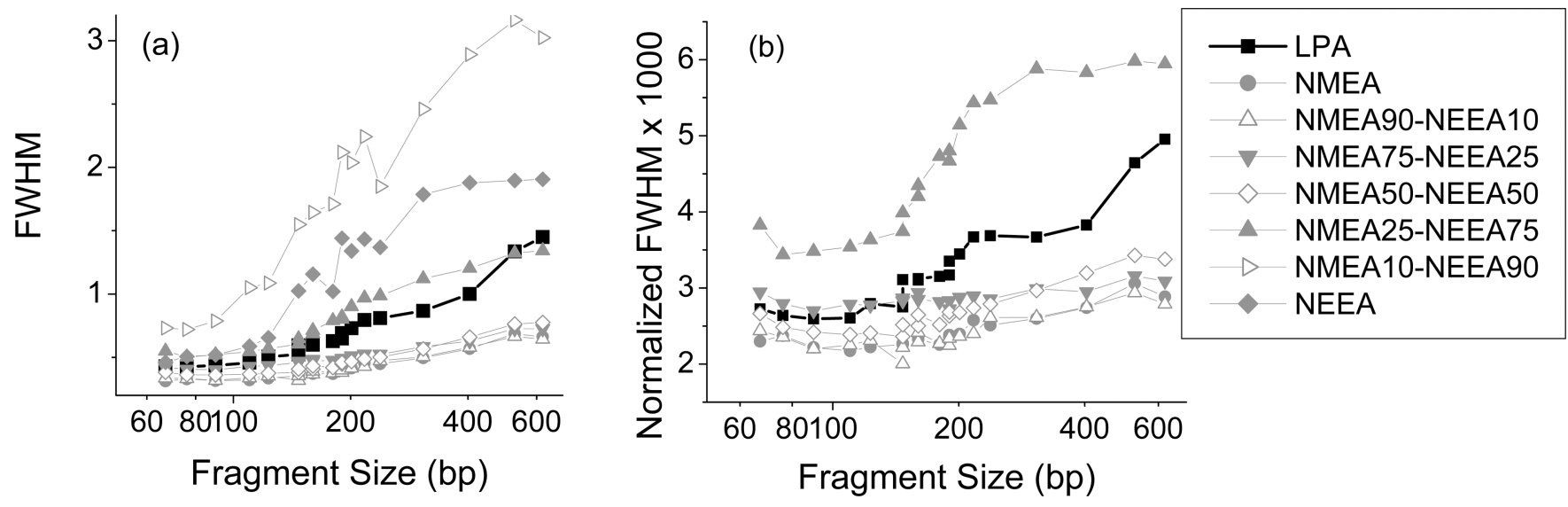

Figure 6.

(a) Full-width at half-maximum (FWHM) and (b) FWHM normalized to the peak migration time of separations performed in 5\% (w/w) solutions at 25 C. NMEA10-NEEA90 and NEEA not shown in (b). $(n=3)$ 


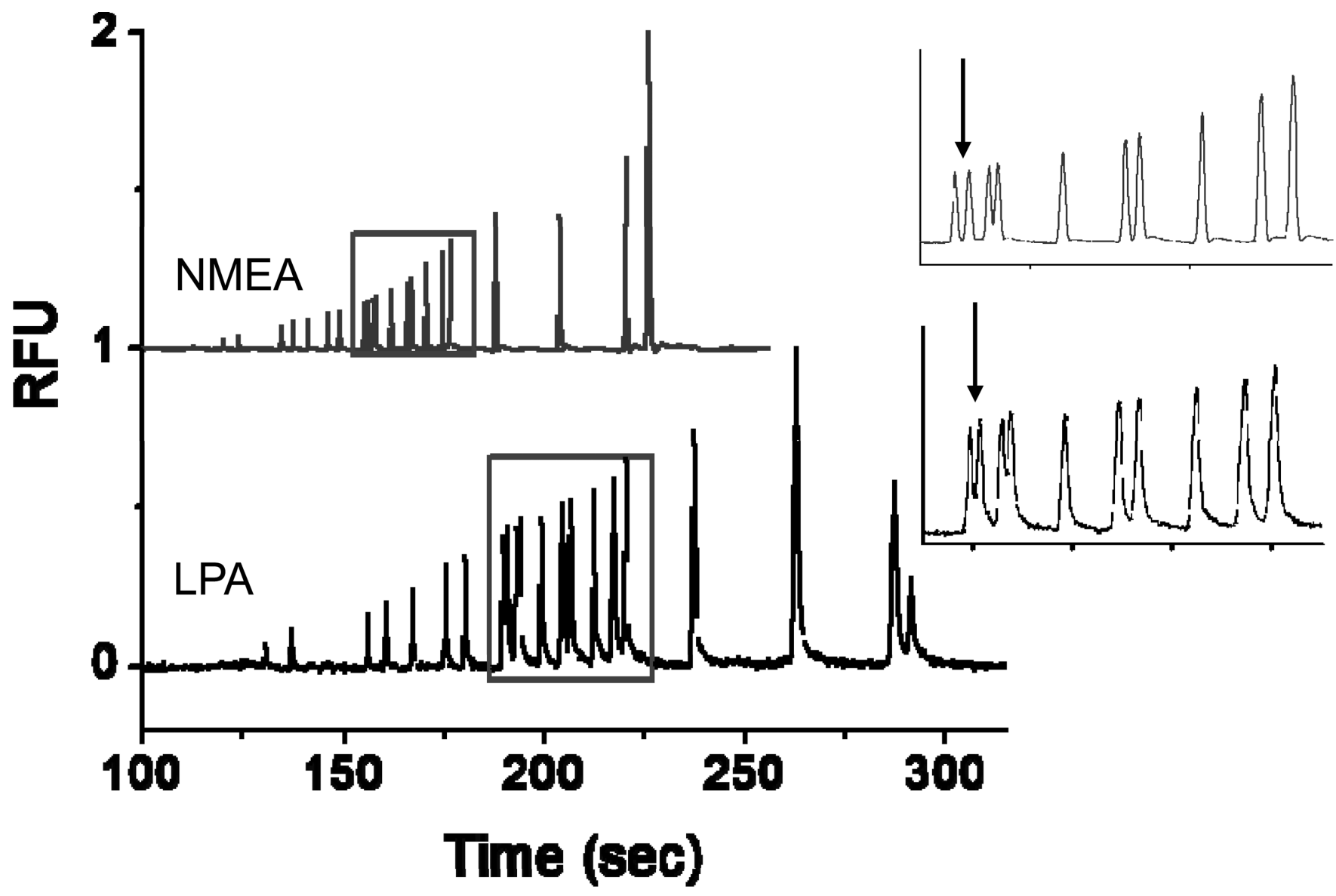

Figure 7.

Microchip separations of pBR322-MspI using a 5\% (w/w) NMEA (top, red) and 5\% (w/w) LPA (bottom, black) at $25^{\circ} \mathrm{C}$. Expanded view highlights improved separation using NMEA as the sieving matrix. 


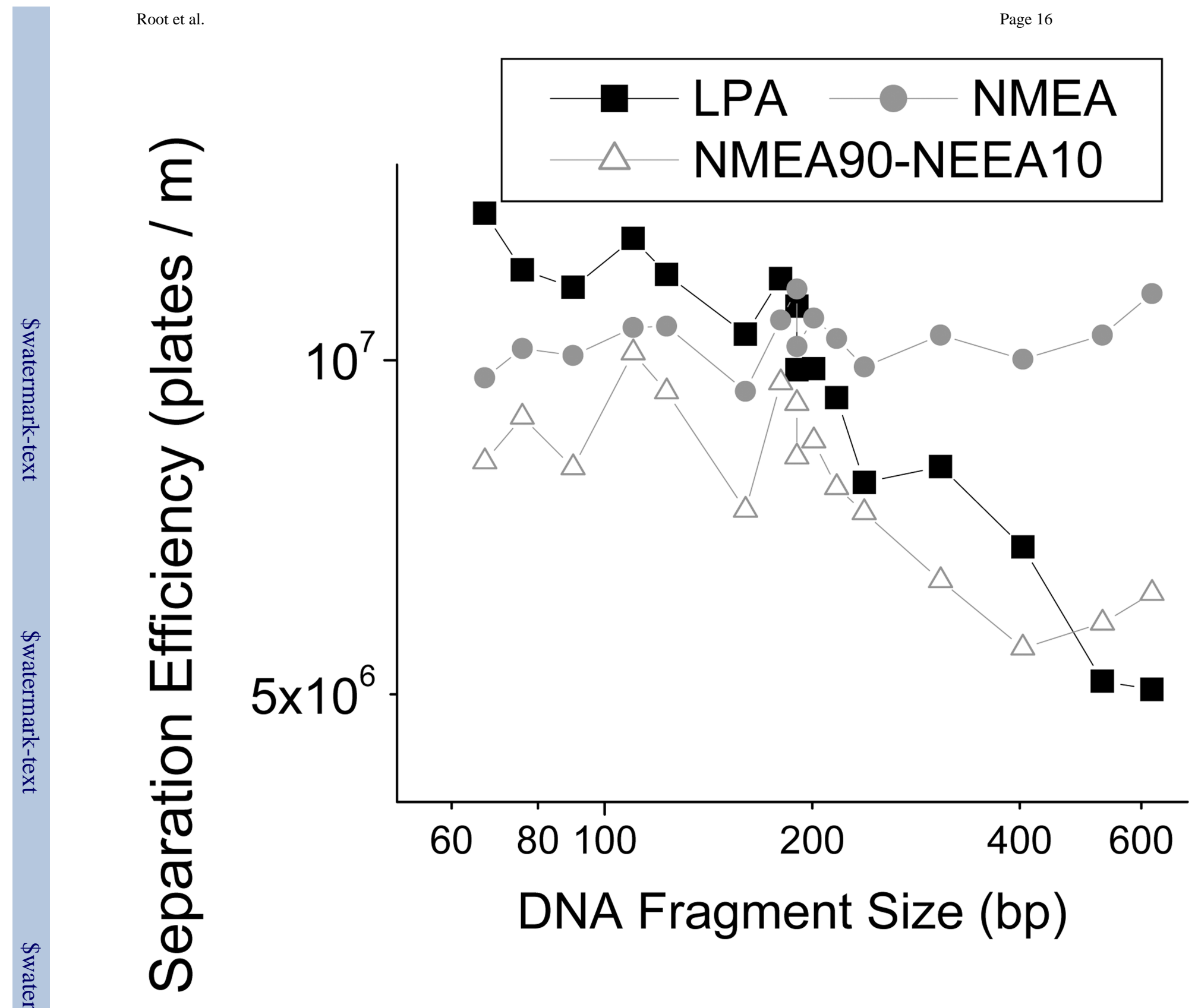

Figure 8.

Average separation efficiency of $5 \%(w / w)$ polymer solutions at $50{ }^{\circ} \mathrm{C}$. $(n=3)$ 


\section{Table 1}

Polymer Molar Masses

\begin{tabular}{lccc} 
Polymer & $\mathbf{M}_{\mathbf{w}}\left(\mathbf{1 0}^{\mathbf{6}} \mathbf{g} / \mathbf{m o l}\right)$ & PDI & $\mathbf{R}_{\mathbf{z}}(\mathbf{n m})$ \\
\hline NMEA & 2.4 & 2.6 & 82 \\
NMEA90 - NEEA10 & 2.1 & 2.7 & 82 \\
NMEA75 - NEEA25 & 2.0 & 2.7 & 81 \\
NMEA50 - NEEA50 & 2.2 & 2.6 & 82 \\
NMEA25 - NEEA75 & 2.1 & 3.2 & 71 \\
NMEA10 - NEEA90 & 2.6 & 2.4 & 55 \\
NEEA & 2.1 & 1.8 & 51 \\
LPA & 2.2 & 2.6 & 83 \\
\hline
\end{tabular}

